\title{
Importance of electronic self-consistency in the TDDFT based treatment of nonadiabatic molecular dynamics
}

\author{
T.A. Niehaus, ${ }^{1}$ D. Heringer, ${ }^{1}$ B. Torralva, ${ }^{2}$ and Th. Frauenheim ${ }^{1}$ \\ 1 Dept. of Theoretical Physics, University of Paderborn, D - 33098 Paderborn, Germany \\ ${ }^{2}$ Chemistry and Materials Science, Lawrence Livermore National Laboratory, Livermore, CA 94550, USA
}

(Dated: August 14, 2018)

\begin{abstract}
A mixed quantum-classical approach to simulate the coupled dynamics of electrons and nuclei in nanoscale molecular systems is presented. The method relies on a second order expansion of the Lagrangian in time-dependent density functional theory (TDDFT) around a suitable reference density. We show that the inclusion of the second order term renders the method a self-consistent scheme and improves the calculated optical spectra of molecules by a proper treatment of the coupled response. In the application to ion-fullerene collisions, the inclusion of self-consistency is found to be crucial for a correct description of the charge transfer between projectile and target. For a model of the photoreceptor in retinal proteins, nonadiabatic molecular dynamics simulations are performed and reveal problems of TDDFT in the prediction of intra-molecular charge transfer excitations.
\end{abstract}

\section{INTRODUCTION}

Beginning with the work of Zangwill and Soven [1], the generalization of density functional theory to time dependent phenomena (TDDFT) has become an important tool in the description of laser-matter interaction. The possible applications are diverse and range from the calculation of spectra (linear optical 2, 3, 4], circular dichroism [5, 6], resonant Raman [7]) to the evaluation of properties (polarization [8, 9], hyperpolarization [10]) up to studies of high harmonic generation 11, 12, 13. and photochemical reactions 14]. The formal justification of TDDFT was laid by Runge and Gross [15], who showed that the exact many body electron density can be obtained from single-particle mean field equations. The solution of these time dependent Kohn-Sham (TDKS) equations can be obtained either perturbatively in the small amplitude limit 16 or by direct numerical integration in the time domain [17]. Both approaches have their inherent merits and disadvantages.

In the linear response regime, for example, the problem can be recast in an eigenvalue equation in the particlehole representation. This allows for an interpretation of optical spectra in terms of contributing single-particle transitions and also for a symmetry assignment of the states. Moreover, transitions with vanishing oscillator strength can be located, like dark singlet or generally triplet states 61]. One of the drawbacks of this approach is the rather poor numerical performance with a scaling of $N^{6}$, where $\mathrm{N}$ is the number of electrons. It should be noted, however, that the CPU time as well as the memory demand can be significantly reduced when iterative procedures like the Davidson algorithm are employed.

In terms of scaling behavior, the numerical integration of the TDKS equations is much more favorable. Here, only the set of occupied orbitals needs to be treated. Because the propagation involves only matrix-vector products, linear scaling can be achieved for large systems [19]. Moreover, since this approach is not restricted to small intensities, non-linear effects like harmonic generation or multiphoton processes can be addressed. Another advantage of working in the real time domain is the possibility to study systematically the effect of different pulse shapes of the laser field on observables such as ionization [20]. With todays femtosecond laser sources, this is currently an active field of experimental research 21].

Nearly all first principles applications of the real time approach have been limited to systems with fixed nuclei. Clearly, it would be highly desirable to study the motion of the coupled system of electrons and nuclei, which would allow one to address problems like laser induced vibrational excitation or photochemical reactions. Since the time step of such molecular dynamics simulations is set to attoseconds by the ultrafast electronic motion, only small systems with a few degrees of freedom can be treated in an ab initio frame work [13, 20]. Consequently, approximate TDDFT methods are quite successful in this domain of application.

Different groups contributed to this field and used their developments in a variety of different studies 14, 22, 23, 24, 25, 26, 27, 28, 29, 30, 31, 32, 33, 34, 35, 36, 37, 39]. In all these approximate schemes only the valence electrons are treated explicitely and the TDKS orbitals are expanded in a limited (usually minimal) basis of atomic orbitals. The Lagrangian, which is a functional of the time dependent density, is then expanded around a static reference density up to a certain order. In zeroth order the Hamiltonian depends only on the reference density, which permits the calculation of the necessary matrix elements once and for all. In this respect, the methods are similar to tight-binding approaches, although no fitting to experimental data is performed.

The purpose of this work is to analyze the implications of extending the mentioned expansion, since all studies so far were restricted to zeroth order. After a more detailed description of the problem in Sec. [II we test the extension in the determination of optical spectra in Sec. IIIA as well as for nonadiabatic molecular dynamics in Sec. IIIB Finally, we perform an investigation of the photochemical reaction of a retinal analogue, 
a chromophore which exhibits an ultrafast radiationless deactivation in nature. These applications in quite different areas of molecular physics are intended to investigate the transferability of the method and also to illustrate the possibilities offered by an approximate solution of the TDDFT equations.

\section{METHOD}

In order to study the dynamics of a coupled system of electrons and nuclei, the equations of motion (EOM) need to be determined. While the electronic EOM in the framework of DFT is given by the well known time dependent Kohn-Sham equations, the nuclear EOM or force equation is not a priori evident. It can be derived either by exploiting the fact that the total energy is a conserved quantity, or by applying the Lagrange formalism. We follow the latter approach here and define the following Lagrangian, which depends on the TDKS states $\Psi_{i}(\mathbf{r} t)$ and the nuclear positions $\mathbf{R}_{A}$ :

$$
\begin{aligned}
\mathcal{L}= & \sum_{A} \frac{1}{2} M_{A} \dot{\mathbf{R}}_{A}^{2} \\
& -\sum_{i}^{\text {occ }}\left\langle\Psi_{i}(\mathbf{r} t)\left|H[\rho](\mathbf{r} t)-i \frac{\partial}{\partial t}\right| \Psi_{i}(\mathbf{r} t)\right\rangle-E_{\mathrm{DC}}-E_{i i},
\end{aligned}
$$

with $\rho(\mathbf{r} t)=\sum_{i}\left|\Psi_{i}(\mathbf{r} t)\right|^{2}$. Here the first term is the classical kinetic energy of the ions, while the remaining terms in Eq. (11) can be obtained from the TDDFT action functional under the assumption that the exchangecorrelation (xc) contributions are local in time [44]. In this widely used adiabatic local density approximation, standard ground state functionals can also be used in the time dependent context simply by evaluation at the time dependent density. Thus, the Hamiltonian $H[\rho](\mathbf{r} t)$ in Eq. (11) takes the common DFT form. Furthermore, $E_{\mathrm{DC}}$ represents the double counting terms

$$
E_{\mathrm{DC}}=-\frac{1}{2} \iint^{\prime} \frac{\rho(\mathbf{r} t) \rho\left(\mathbf{r}^{\prime} t\right)}{\left|\mathbf{r}-\mathbf{r}^{\prime}\right|}+E_{x c}[\rho]-\int v_{x c}[\rho] \rho(\mathbf{r} t),
$$

and $E_{i i}$ the ion-ion repulsion (Here and in the following $\int d \mathbf{r}^{\prime}$ is abbreviated as $\int^{\prime}$, and $\int d \mathbf{r}$ as $\left.\int\right)$.

We now proceed by applying the same kind of approximations as were used in the derivation of the density functional theory based tight-binding (DFTB) method [40, 41] from static DFT. To keep the presentation concise we refer to some reviews [38, 39], which provide a more detailed description of the basic concepts, practical realization and accuracy of the ground state DFTB approach. Here, we only report aspects, which are specific for the generalization to the time-dependent case. In a first step, the Lagrangian is expanded around a reference density $\rho_{0}(\mathbf{r}), \rho(\mathbf{r} t)=\rho_{0}(\mathbf{r})+\delta \rho(\mathbf{r} t)$, which is given as a superposition of atomic (ground state) densities. In contrast to our earlier work [39], we now include terms up to second order in the density fluctuations $\delta \rho(\mathbf{r} t)$ :

$$
\begin{array}{r}
\mathcal{L} \approx \sum_{A} \frac{1}{2} M_{A} \dot{\mathbf{R}}_{A}^{2}-\sum_{i}^{\text {occ }}\left\langle\Psi_{i}(\mathbf{r} t)\left|H\left[\rho_{0}\right](\mathbf{r})-i \frac{\partial}{\partial t}\right| \Psi_{i}(\mathbf{r} t)\right\rangle \\
+\frac{1}{2} \iint^{\prime} \frac{\rho_{0}(\mathbf{r}) \rho_{0}\left(\mathbf{r}^{\prime}\right)}{\left|\mathbf{r}-\mathbf{r}^{\prime}\right|}-E_{x c}\left[\rho_{0}\right]+\int v_{x c}\left[\rho_{0}\right] \rho_{0}(\mathbf{r})-E_{i i}
\end{array}
$$

$$
-\frac{1}{2} \iint^{\prime}\left(\frac{1}{\left|\mathbf{r}-\mathbf{r}^{\prime}\right|}+\frac{\delta v_{x c}[\rho](\mathbf{r} t)}{\delta \rho\left(\mathbf{r}^{\prime} t\right)}\right) \delta \rho(\mathbf{r} t) \delta \rho\left(\mathbf{r}^{\prime} t\right) .
$$

Please note that in this expansion all contributions which are linear in $\delta \rho$ are captured by the second term in Eq. (3a) through the TDKS states. The terms in Eq. (3b) can now be subsumed as $E_{\text {rep }}$, a sum of short ranged pair potentials, which depend only on the atomic species and the interatomic distance. Since $E_{\text {rep }}$ is a functional of the time independent reference density $\rho_{0}$ only, it is exactly the same as used in the ground state DFTB scheme. The second order term of Eq. (3C), which is the focus of this work, is approximated as follows:

$$
\begin{aligned}
E_{2 \text { nd }} & =\frac{1}{2} \iint^{\prime}\left(\frac{1}{\left|\mathbf{r}-\mathbf{r}^{\prime}\right|}+\frac{\delta v_{x c}[\rho](\mathbf{r} t)}{\delta \rho\left(\mathbf{r}^{\prime} t\right)}\right) \delta \rho(\mathbf{r} t) \delta \rho\left(\mathbf{r}^{\prime} t\right) \\
& \approx \frac{1}{2} \sum_{A B} \Delta q_{A}(t) \gamma_{A B} \Delta q_{B}(t) .
\end{aligned}
$$

Here the $\Delta q_{A}(t)$ denote atomic net Mulliken charges

$$
\begin{aligned}
\Delta q_{A}(t) & =q_{A}(t)-q_{A}^{\text {free atom }} \\
q_{A}(t) & =\frac{1}{2} \sum_{i} \sum_{\mu \in A, \nu}\left(b_{\mu i}^{*}(t) b_{\nu i}(t) S_{\mu \nu}+b_{\nu i}^{*}(t) b_{\mu i}(t) S_{\nu \mu}\right),
\end{aligned}
$$

where the coefficients $b_{\mu i}(t)$ are defined by the expansion of the TDKS states in a basis of non-orthogonal atomic orbitals $\phi_{\mu}\left(\mathbf{r}-\mathbf{R}_{A}\right)$

$$
\Psi_{i}(\mathbf{r} t)=\sum_{\mu} b_{\mu i}(t) \phi_{\mu}\left(\mathbf{r}-\mathbf{R}_{A}\right),
$$

which build the overlap matrix $S_{\mu \nu}=\left\langle\phi_{\mu} \mid \phi_{\nu}\right\rangle$. Further, the function $\gamma_{A B}$ in Eq. 团 interpolates between a pure Coulomb interaction for large interatomic distances and an element-specific constant in the atomic limit. This numerically evaluated parameter includes the effects of exchange and correlation and is directly related to the chemical hardness of the atomic species [41]. Taking the coefficients and nuclear positions as generalized coordinates, the evaluation of the Euler-Lagrange equations leads to the desired equations of motion. The electronic motion obeys:

$$
\dot{b}_{\nu i}=-\sum_{\delta \mu} S_{\nu \delta}^{-1}\left[i H_{\delta \mu}+\sum_{A} \dot{\mathbf{R}}_{A}\left\langle\phi_{\delta} \mid \frac{\partial}{\partial \mathbf{R}_{A}} \phi_{\mu}\right\rangle\right] b_{\mu i}
$$

with

$$
\begin{aligned}
H_{\mu \nu} & =\left\langle\phi_{\mu}\left|H\left[\rho_{0}\right]\right| \phi_{\nu}\right\rangle+\frac{1}{2} S_{\mu \nu} \sum_{C}\left(\gamma_{A C}+\gamma_{B C}\right) \Delta q_{C} . \\
& =H_{\mu \nu}^{0}+H_{\mu \nu}^{1} ; \quad \forall \mu \in A ; \nu \in B .
\end{aligned}
$$


In zeroth order the Hamiltonian reduces to the first term in Eq. (7b) and depends solely on the reference density $\rho_{0}$. For systems in the excited state or in charged or heteronuclear structures, the electronic density differs significantly from a simple superposition of atomic ground state densities. To a certain extent this difference is already captured at the zeroth order level, since the coefficients which solve Eq. (7a) correspond to a timedependent density different from $\rho_{0}$. This is similar to the situation in empirical tight-binding schemes for the ground state, where even certain ionic crystals are suffciently well described [42]. However, a consideration of the full Hamiltonian in Eq. (7b) leads obviously to a more balanced treatment, because dynamical changes in the electron density are explicitly included in a selfconsistent fashion. In this way, one can even hope to correctly describe the large amplitude motion induced by intense laser fields.

Solution of Eq. (7a) requires an iterative procedure with timesteps in the attosecond regime. A symplectic algorithm is used for this task 37], which is based on the Cayley representation of the time evolution operator and conserves the norm of the wavefunction exactly. Besides the Hamiltonian and overlap matrices (see Ref. [39] for details of the construction), Eq. (7a) contains also the nonadiabatic coupling matrix $\left\langle\phi_{\mu} \mid \frac{\partial}{\partial \mathbf{R}_{A}} \phi_{\nu}\right\rangle$, in which all on-site elements $\left(\phi_{\mu}, \phi_{\nu}\right.$ on the same atom) are set to zero [43. This allows one to relate the remaining elements to a simple derivative of the overlap matrix.

Variation of the Lagrangian with respect to the nuclear coordinates leads to the following expression for the forces:

$$
\begin{aligned}
M_{A} \ddot{\mathbf{R}}_{A}= & -\sum_{i}^{\text {occ }} \sum_{\mu \nu} b_{\mu i}^{*} b_{\nu i}\left(\frac{d H_{\mu \nu}^{0}}{d \mathbf{R}_{A}}+\frac{d S_{\mu \nu}}{d \mathbf{R}_{A}} \sum_{B} \gamma_{A B} \Delta q_{B}\right) \\
& +\frac{1}{2} \sum_{i}^{\text {occ }} \sum_{\mu \nu \delta \gamma}\left(b_{\mu i}^{*} \frac{d S_{\mu \nu}}{d \mathbf{R}_{A}} S_{\nu \delta}^{-1} H_{\delta \gamma} b_{\gamma i}+\text { c.c. }\right) \\
& -\Delta q_{A} \sum_{B} \frac{d \gamma_{A B}}{d \mathbf{R}_{A}} \Delta q_{B}-\frac{d E_{\mathrm{rep}}}{d \mathbf{R}_{A}} .
\end{aligned}
$$

Since the Hamiltonian is time dependent due to an external field or nuclear motion, the molecular orbital coefficients will in general represent a coherent superposition of different eigenstates of the system. In this case, the nuclei move in a mean potential according to Eq. 8 rather than being restricted to a particular Born-Oppenheimer surface as in conventional adiabatic MD approaches. In fact, due to the coupling of the EOM, energy can be freely exchanged between the electronic and ionic subsystems as long as the total energy of the system is conserved. Equations of motion that are equivalent to the ones reported here, have been derived earlier by Saalmann and Schmidt [29] as well as Todorov [36]. Including the second order correction, they are solved here for the first time in an actual calculation.

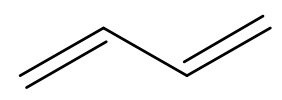

FIG. 1: Schematic illustration of trans-butadiene $\left(\mathrm{C}_{4} \mathrm{H}_{6}\right)$.

Todorov pointed out, that for an incomplete basis the force equation has to be augmented by additional velocity dependent terms, which should become important in high energy collisions. Interestingly, omission of these terms does not violate energy but only momentum conservation. Hence, it is useful to monitor the total momentum (electronic + ionic) of the system if Eq. (8) is used, as in every practical calculation the basis set is incomplete.

Finally, in order to simulate the interaction with electromagnetic fields, the vector potential $\mathbf{A}(\mathbf{r} t)$ needs to be incorporated, which is done via minimal coupling, $\mathbf{p}-\frac{e}{c} \mathbf{A}$. A numerically efficient approximation was proposed by Graf and Vogl [45] and later by Allen 22] and is given by:

$$
\begin{aligned}
H_{\mu \nu}[\mathbf{r}, \mathbf{p}, \mathbf{A}(t)]= & \exp \left[\frac{i e}{\hbar c}\left(\mathbf{R}_{A}-\mathbf{R}_{B}\right) \mathbf{A}(t)\right] \\
& \times H_{\mu \nu}[\mathbf{r}, \mathbf{p}] ; \quad \mu \in A, \nu \in B,
\end{aligned}
$$

which relates the desired field dependent Hamiltonian to the already known matrix elements of the unperturbed one. Expression (9) was derived under the assumption that the radiation wavelength is much larger than the molecular system under study, which is usually well fulfilled for frequencies in the optical range. It should be mentioned, that Eq. (9) in principle holds for arbitrarily strong fields in contrast to the electric dipole approximation.

If the interest is just on calculating optical spectra, rather than the molecular motion initiated by a laser pulse, only Eq. (7a) needs to be solved for a fixed geometry. Following the approach of Yabana and Bertsch [17, 46] the field in Eq. (9) is turned on only at a certain instant of time, which populates the complete manifold of excited states. The time dependent dipole moment $d(t)$ can then be used to calculate the dynamic polarizability:

$$
\alpha(\omega)=\frac{\hbar c}{e A} \int e^{i \omega t}(d(t)-d(0)) d t
$$

and the dipole strength function $S(\omega)=2 \omega / \pi \Im \alpha(\omega)$ of the system; a quantity which can be directly compared to experimental spectra.

\section{APPLICATIONS}

\section{A. Optical spectrum of trans-butadiene}

As a first application of our method, we examine a prototypical $\pi$-system, trans-butadiene (Fig. (1). The optical 


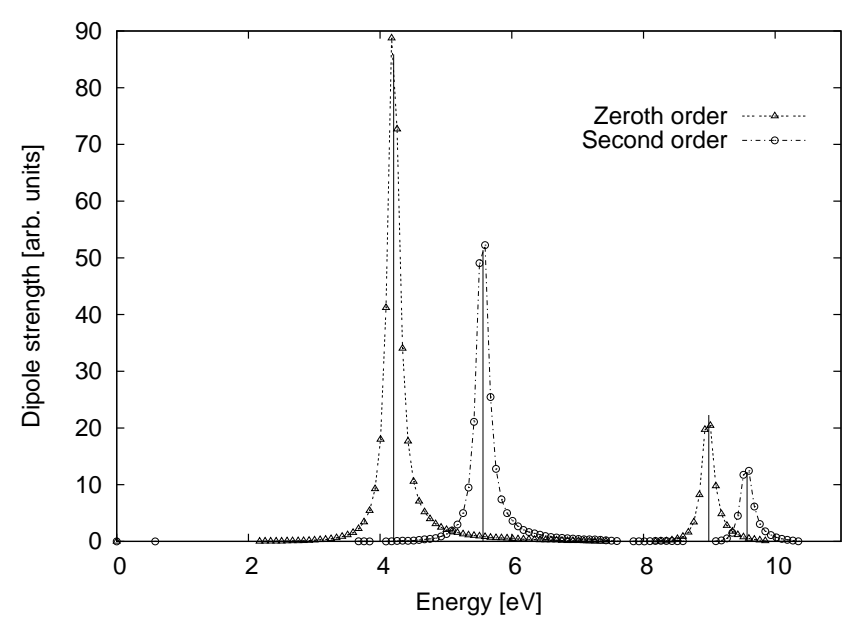

FIG. 2: Dipole strength of trans-butadiene as given by the time-dependent DFTB method in zeroth and second order [47]. Shown is an average over different molecular orientations with respect to the polarization of the vector potential. Results obtained in the DFTB linear-response implementation of TDDFT are shown as stick spectrum. The associated oscillator strengths have been normalized to the maximum of the highest energy peak.

spectrum of this molecule has been the subject of numerous quantum chemical studies (see e.g. Ref. 48 and references therein). Recently, also a detailed investigation of the molecular dynamics in the excited state appeared [14. Dou et al. employed the DFTB method described in this work without the second order correction. Our interest here is to analyze the implications of including this term. To this end, we first relaxed the molecule with the ground state DFTB method and recorded the optical spectrum according to the prescription given in Sec. III After applying a vector potential of $\mathrm{A}=0.0125$ gauss $\mathrm{cm}$, the Kohn-Sham orbitals were propagated for $38.7 \mathrm{fs}$ with a time step of 12 as. Since the finite sampling introduces spurious negative parts in the imaginary part of the polarizability, the dipole moment was damped with a factor of $e^{-k t}(\mathrm{k}=0.3 \mathrm{eV} / \hbar)$, like in Ref. 17. This also simulates dephasing or other line broadening effects which would appear more naturally in a more complete theory. The resulting spectrum with and without second order correction is shown in Fig. 2. As can be seen, the maximum absorption in the latter method is located at 4.21 $\mathrm{eV}$. This is exactly the difference of the LUMO (lowest unoccupied molecular orbital) and the HOMO (highest occupied molecular orbital) energies of the ground state DFTB method. If the second order term is included in the calculation, the absorption is strongly blue shifted to $5.56 \mathrm{eV}$, which is in good agreement with the experimental value of $5.8 \mathrm{eV}$ [4]. Along with the energy shift, a reduction of absorption strength is also observed.

To better understand the origin of these changes, we also performed calculations with our implementation of the TDDFT linear response formalism [50]. In this ap- proach, excited state singlet energies $\omega_{I}$ are given by the solution of the following eigenvalue problem:

$$
\sum_{k l}\left[\omega_{i j}^{2} \delta_{i k} \delta_{j l}+2 \sqrt{\omega_{i j}} K_{i j, k l} \sqrt{\omega_{k l}}\right] F_{k l}^{I}=\omega_{I}^{2} F_{i j}^{I} .
$$

Here the $\omega_{i j}$ are energy differences between unoccupied orbitals $j$ and occupied orbitals $i$, while the so called coupling matrix $K$ describes the change of the SCF potential due to the induced density. As Eq. (11) shows, the effect of the coupling matrix is not only to shift the true excited state away from simple orbital energy differences, but also to couple different single-particle transitions. The explicit form of $K$ is given by:

$$
\begin{aligned}
K_{i j, k l}= & \iint^{\prime} \psi_{i}(\mathbf{r}) \psi_{j}(\mathbf{r}) \\
& \times\left(\frac{1}{\left|\mathbf{r}-\mathbf{r}^{\prime}\right|}+\frac{\delta v_{x c}[\rho](\mathbf{r})}{\delta \rho\left(\mathbf{r}^{\prime}\right)}\right) \psi_{k}\left(\mathbf{r}^{\prime}\right) \psi_{l}\left(\mathbf{r}^{\prime}\right)
\end{aligned}
$$

which is nothing else than the second order term of Eq. (3c), when the induced density is expanded in particle-hole states. The results of the linear response calculations with and without the coupling matrix contribution are given as stick spectrum in Fig. 1] Obviously, there is a perfect match beween the real time and linear response approaches to TDDFT both in the energetical position of the states and the oscillator strength. This equivalence had to be expected, since the linear response approach amounts to a perturbative solution of the TDDFT equations in the small amplitude limit. However, to our knowledge this has so far never been shown in practical calculations.

\section{B. $\mathrm{C}^{+}-\mathrm{C}_{60}$ collisions}

Ion-cluster collisions provide an ideal application field for approximate TDDFT molecular dynamics simulations. This is because the number of degrees of freedom is usually too large to be treated with first principles calculations and also nonadiabatic effects are strong and important. Depending on the velocity of the projectile, collisions can induce vibrational or a combination of electronic and vibrational excitations of the cluster, where the latter type cannot be described in conventional Born-Oppenheimer dynamics. In this context, Kunert and Schmidt undertook a systematic investigation of ion-fullerene collisions and provided an explanation for seemingly conflicting experimental observations [34]. Their nonadiabatic quantum molecular dynamics (NA-QMD) method is essentially equivalent to the DFTB scheme described here in the zeroth order approximation. Accordingly, it is interesting to see whether the second order correction is of any benefit in these kind of simulations.

For the special case of $\mathrm{C}^{+}-\mathrm{C}_{60}$ collisions we performed calculations for different values of the impact velocity $(v$ $=0.01 \ldots 0.45$ a.u. $)$ and impact parameter $(b=2.0,7.5$ 


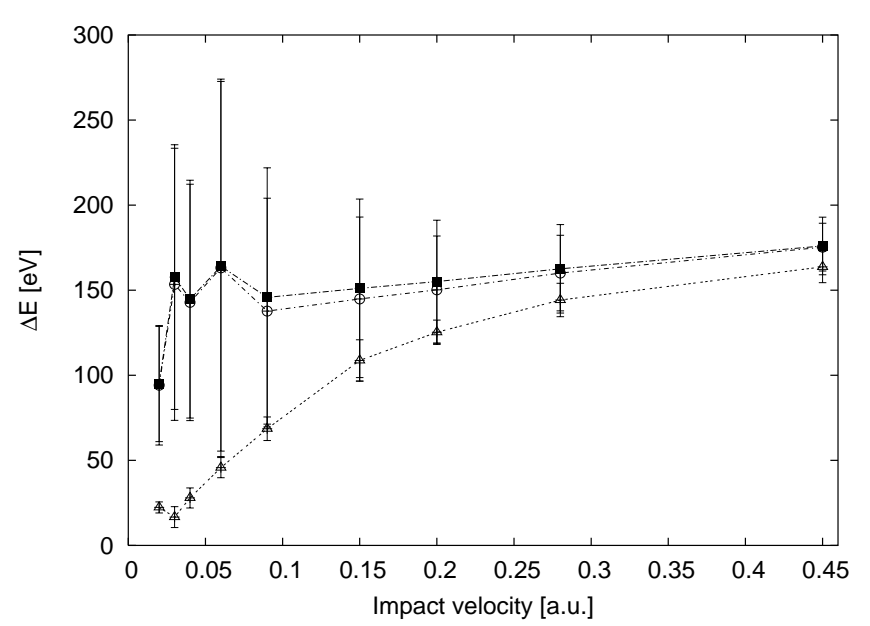

FIG. 3: Total kinetic energy loss $\Delta E$ of the projectile in the center of mass system for an impact parameter of $b=2.0$ a.u. For each velocity three trajectories with random cage orientation were calculated; error bars correspond to the standard deviation. Dark squares: second order, open circles: zeroth order DFTB results, open triangles: loss due to electronic excitation, obtained from the difference of time-dependent and ground state energy in the zeroth order DFTB scheme.

a.u.) for randomly oriented fullerene cages. Special care had to be taken in the definition of the initial conditions of the EOM, since the $\mathrm{C}^{+}-\mathrm{C}_{60}$ configuration does not correspond to the ground state of the system. For that reason, we performed separate ground state calculations for the two subsystems and combined the resulting KS orbitals to obtain the desired charge state. The initial ion-cluster distance was chosen large enough to prevent any interaction and the system was then left to evolve freely according to the EOM [Eq. (7a) and (8)].

Fig. 3 depicts the total kinetic energy loss $\Delta E$ in the center of mass system that the projectile experiences due to the collision. It can be directly compared to the results in Fig. 3 of Ref. 34, which were obtained for a single fixed collision geometry. As already shown there, the vibrational excitation of the fullerene dominates for smaller velocities $(v<0.1$ a.u. $)$, while mostly electronic excitation is responsible for the energy loss at larger impact energies. As the impact velocity increases, $\Delta E$ first rises, peaks around 0.05 a.u and shows a weak increase beyond 0.1 a.u. in our calculations. This is in variance with the results of Kunert and Schmidt [34], which claim velocity-independent excitation energies in the high velocity range. Taking the strong dependence on the collision geometry into account, an extensive phase space sampling would be necessary to resolve this issue, which is outside the scope of this work.

Turning now to a comparison of the predictions of DFTB with and without second order correction, we find only marginal differences in the results of both methods. Such a difference could have been expected for high impact velocities, but with such a large amount of energy

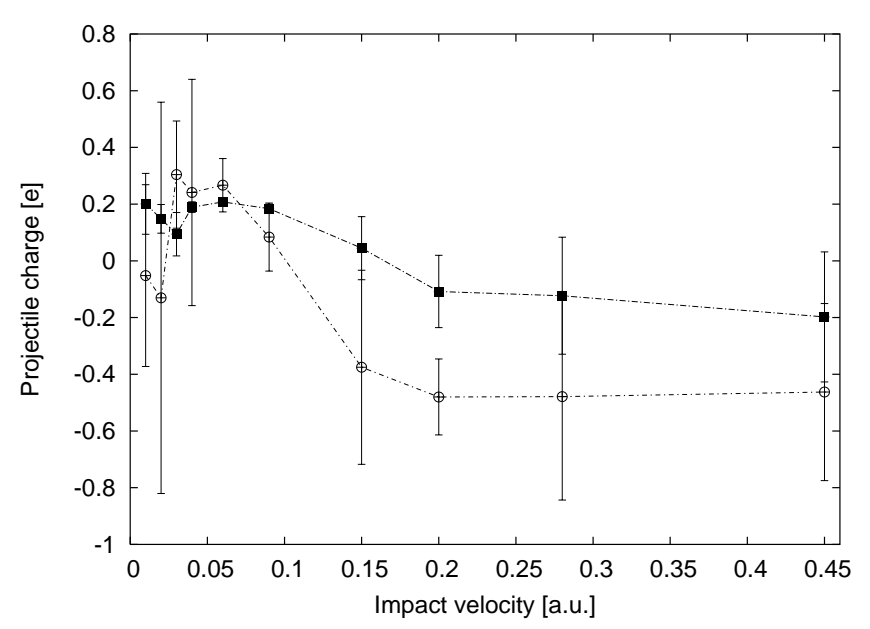

FIG. 4: Charge of the carbon atom after the collision with $\mathrm{C}_{60}$ for different values of the impact velocity and an impact parameter of $b=7.5$ a.u. . For each velocity three trajectories with random cage orientation were calculated; error bars correspond to the standard deviation. Dark squares: second order, open circles: zeroth order DFTB results.

deposited in the cluster, finer details of the electronic structure seem to have negligible influence.

The second order correction has however implications for other observables. Fig. 4 shows the charge of the projectile after the collision. Here fractional charges need to be understood in the probabilistic interpretation of quantum mechanics, since in the simulations the system remains in a superposition of eigenstates with integer charges also asymptotically. For higher velocities, which correspond to higher electronic excitation as mentioned above, the charge given by the zeroth order DFTB method is significantly more negative than the second order one. This can be explained by a larger contribution of the asymptotic $\mathrm{C}^{-}-\mathrm{C}_{60}^{2+}$ state, which in the zeroth order approximation is located only slightly higher in energy as the initial $\mathrm{C}^{+}-\mathrm{C}_{60}^{0}$ state. As the results in Tab. show, this energy ordering is in striking contrast with the one given by the second order DFTB method and experiment. Although calculations of charge transfer cross sections have been performed in a zeroth order scheme [32, 33], the results of this section suggest, that in general a more advanced treatment is absolutely necessary.

\section{Protonated Schiff base photodynamics}

As a last application we study the photodynamics of the retinal molecule, which is of special importance in the field of biology. This chromophore is found in a variety of proteins, where it initiates quite different reactions in the cell. In bacteriorhodopsin and halorhodopsin for example, light absorption of retinal triggers the membrane transport of protons and chloride ions, respectively. In contrast, it starts a cascade of reactions that initiate the 
TABLE I: Energy ordering of different asymptotic states of the singly positively charged $\mathrm{C}-\mathrm{C}_{60}$ system in $\mathrm{eV}$. The DFTB energies with and without second order correction were obtained by separate calculations of the two subsystems and addition of the results. For $\mathrm{C}_{60}$, all calculations were performed at the DFTB optimized geometry of the neutral species. The experimental results were obtained from measured ionization potentials and electron affinities [51, 52, 53]. The most stable state of each method was set to zero energy.

\begin{tabular}{ccccc}
\hline \hline $\mathrm{C}$ & $\mathrm{C}_{60}$ & zeroth order & second order & exp. \\
\hline+ & 0 & 0.0 & 2.68 & 3.66 \\
0 & + & 0.58 & 0.0 & 0.0 \\
- & $2+$ & 1.16 & 10.80 & $7.14 \ldots 9.14$ \\
\hline \hline
\end{tabular}



FIG. 5: Top: Structure of 11-cis retinal, which is found in dark adapted rhodopsin and transforms to the all-trans form upon absorption of light. Bottom: Protonated Schiff base model used in this study.

vision process in rhodopsin. In all these systems, the retinal is known to isomerize around a specific double bond. The quantum yield of the photoreaction is particularly high $(\Phi \approx 0.7)$ and the deexcitation to the ground state occurs in no more than 200-500 fs [54, [55]. Because of these unusual features, this system provides an interesting subject for a theoretical investigation. For a complete understanding of the retinal photodynamics it would certainly be necessary to include the full protein environment in such an investigation. However, important information can already be drawn from the examination of small retinal analogues like the protonated Schiff bases (Fig. [5). These models share a polyene chain of alternating single and double bonds with retinal, as well as the positively charged $\mathrm{NH}_{2}^{+}$Schiff base group, which is crucial for the function of the chromophore in the protein. High level quantum chemical CASPT2 calculations on these analogues revealed, that after absorption the system moves out of the Franck-Condon region along the $\mathrm{C}=\mathrm{C}$ stretch normal coordinate (see Ref. 56 and references therein). After inversion of single and double bonds, torsion around the central $\mathrm{C}=\mathrm{C}$ bond sets in. A barrierless path then leads to a conical intersection of ground and excited state, where efficient deactivation to the photoproduct occurs. In line with Stark spectroscopy
[57, 58], CASPT2 theory predicts a large charge transfer from the Schiff base group to the other terminus upon excitation, that increases along the excited state pathway.

Recently, we investigated the excited state potential energy surface (PES) obtained from static DFTB calculations in the linear response formalism and found severe deviations from the CASPT2 results [59]. In fact, the only barrierless paths found to conical intersections with the ground state involved single rather than double bond isomerization (in accordance with ab initio TDDFT calculations). An intrinsic reaction coordinate connecting Franck-Condon point and correct conical intersection (as described in the CASSCF model) includes a significant barrier. Thus an efficient and ultrafast reaction seems to be unlikely at the DFT level of theory. However, one should keep in mind, that at finite temperature molecules posses a significant amount of kinetic energy already at the Franck-Condon point, which allows the system to sample a large fraction of the excited state PES. Hence, the minimum energy path might not necessarily provide a representative description of the photodynamical pathway. Moreover, nonadiabatic transitions are not restricted to conical intersections. They can also occur in regions where there is a finite gap between the ground and excited state surfaces, especially when the atomic velocity is high. Our interest is therefore to perform nonadiabatic molecular dynamics simulations of the PSB model system to see whether the discrepancies between DFT predictions and experiment remain at a full dynamical level.

A complete description of the photochemical process would in principle require a full phase space sampling prior to excitation. Since the maximum absorption is strongly geometry dependent, we nevertheless take only the relaxed geometry of the ground state minimum into account. At the Franck-Condon point random velocities corresponding to a temperature of $300 \mathrm{~K}$ are assigned to the atoms and the system is left to evolve freely without further constraints. The excitation itself is induced by a Gaussian shaped laser pulse with a central frequency of $3.45 \mathrm{eV}$, that is slightly detuned with respect to the maximum absorption to avoid population of higher excited states close in energy. The maximum absorption itself is located at $3.88 \mathrm{eV}$ in the second order DFTB method and agrees well with first principles TDDFT calculations [59] $(4.03 \mathrm{eV})$ as well as CASPT2 [60] $(4.02 \mathrm{eV})$ and experiment $(3.85-4.25 \mathrm{eV}[61])$. Similar to the case of trans-butadiene, the excitation energy is strongly underestimated at $2.66 \mathrm{eV}$, if the second order correction is not taken into account. Since the nonadiabatic excitation process depends strongly on the gap between ground and excited state PES, the DFTB method without electronic selfconsistency will therefore provide an unrealistic description of the photodynamics and will not be used here. The duration and fluence of the laser pulse were chosen to be 5.9 fs and $5.4 \mathrm{~mJ} / \mathrm{cm}^{2}(\mathrm{~A}=0.7$ gauss $\mathrm{cm})$. For these parameters, the total energy of the system rises 




FIG. 6: Bond alternation in the PSB model system for all calculated trajectories, estimated as the mean bond length difference between neighboring $\mathrm{C}-\mathrm{C}$ single and double bonds.

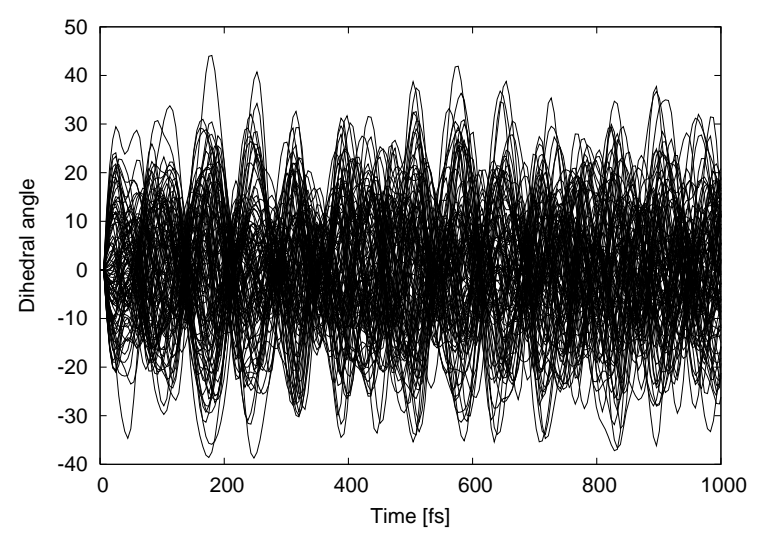

FIG. 7: Torsion angle around the central double bond of the PSB model for all calculated trajectories.

by $3.88 \mathrm{eV}$, i.e. a one-photon transition to the first excited state is simulated. For a total simulation time of $1.1 \mathrm{ps}, 100$ trajectories were propagated with a timestep of 12.1 as. This guaranteed an energy conservation of $\Delta E / E \approx 10^{-8}$. With these parameters, one trajectory took about 22 minutes CPU time on an Intel Xeon 3.06 $\mathrm{GHz}$ processor.

The results of the simulations show that the initial dynamics on the excited PES are dominated by C-C stretch motions with large amplitudes up to $0.15 \AA$. This is in agreement with resonant Raman studies on the PSB in solution and in the rhodopsin protein 63, 64, 65. In contrast to the mentioned CASPT2 calculations however, the bond alternation is only inverted for roughly half of the trajectories as shown in Fig. [6 Moreover, the timeaveraged bond alternation of 0.060 Ås only slightly reduced with respect to the ground state minimum $(0.063$ $\AA$ ). Considering now the dihedral angle which represents the torsion around the central double bond, Fig. 7 shows that none of the 100 trajectories resulted in a successful isomerization within 1 ps. We find much larger amplitudes for the rotation around single bonds, with tor-

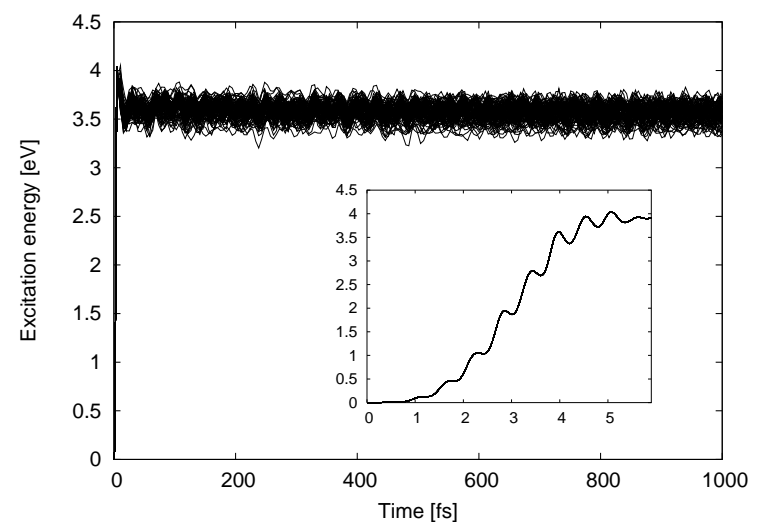

FIG. 8: Excitation energy of the PSB model versus time in $\mathrm{eV}$. The inset shows the transition to the excited state due to the applied laser pulse.

sion angles up to $80^{\circ}$ for certain trajectories, although also here no isomerization is completed in the simulation time. This preference of single over double bond isomerization in DFT based methods was already found in the static investigation of Ref. 59. Another prediction of CASPT2 theory, which is in nice agreement with Stark spectroscopy has already been mentioned. The $S_{0}-S_{1}$ excitation and the subsequent motion on the $S_{1}$ PES is known to involve a large charge transfer away from the Schiff base group. We however, do not observe any significant charge transfer in our simulations.

Finally, it is interesting to analyze whether deexcitation to the ground state occurred. In Fig. 8 the excitation energy of the system is shown, which is given by the difference of the time-dependent and ground state energy at the same geometry. Directly after the end of the laser pulse, a small reduction of the excitation energy is observed $(\approx 0.3 \mathrm{eV})$, which is related to an elongation of all bonds in the PSB model. There is little change from this point on and nonadiabatic transitions, which would manifest in a sharp drop of the excitation energy, do not occur. In our DFT based treatment, deactivation is therefore predicted to happen on longer timescales, presumably involving the slower processes of internal conversion and fluorescence. At any rate, ultrafast isomerization with a concomitant intersection of ground and excited state is not found to be the dominant process. This is in stark contrast to the results of Vreven et al. [6] , who showed that double bond isomerization can occur in less than 100 fs for the model at hand.

The simulations of this section could be extended by computing a larger number of trajectories or a longer propagation time. Considering the narrow distribution of the results presented in Fig. 6 to 8 it is not very likely that an improved sampling would reveal new information. Extension of the simulation time might seem advantageous in light of the experiments by Logunov et al. 67], who measured an excited state life time of 2-3




tion. However, for the shorter PSB model used in this study, the excited state PES has been found to be much steeper [56] in line with the mentioned study of Vreven et al. [66]. Hence, the photochemical process should be completed in the chosen simulation time of 1 ps.

To summarize, we find that our simulations disagree in most aspects with CASPT2 results and experiment. At the same time we confirmed the static investigations of the DFT based potential energy surfaces from Ref. 59 by dynamical calculations. Given that, (i) timedependent DFTB and first principles TDDFT, as well as, (ii) TDDFT with different exchange-correlation functionals (local, gradient corrected or hybrid) yield qualitatively the same picture [59], a correct DFT based description of the retinal photodynamics is highly unlikely. Only very recently, failures of TDDFT in the description of inter-molecular charge transfer states were reported 68]. Exchange-correlation functionals that address this shortcoming have also been recently proposed [69, 70, 71]. It will be interesting to see whether these developments can also remedy the problems with intra-molecular charge transfer found here.

\section{SUMMARY}

In this work, we presented a mixed quantum-classical approach to simulate the coupled dynamics of electrons and nuclei. The method is based on a second order expansion of the TDDFT Lagrangian around a suitable reference density. We showed that the inclusion of the second order term improves both qualitatively and quantitatively the optical spectrum of molecules. For transbutadiene a strong blueshift of the absorption was observed together with a significant reduction of oscillator strength. In this context, the analogy with the linear response approach to TDDFT revealed that the zeroth order treatment of the Lagrangian corresponds to an un- coupled response that neglects collective effects. Moreover, experience with the linear response implementation suggests that this absorption shift is quite general and especially large for $\pi-\pi^{*}$ transitions. For $n-\pi^{*}$ excitations however, the coupling is usually weak and realistic results might already be achieved in the zeroth order approximation. In the simulations of high energy collision of Sec. IIIB there is little difference in the predictions of both schemes when the interest is in energy transfer only. This is because the process is dominated by vibrational rather than electronic excitation in the regime of low energy transfer, where the different level structure could be resolved. Considering now the charge transfer, we found important differences for the $\mathrm{C}^{+}-\mathrm{C}_{60}$ system, which were attributed to the problematic description of the asymptotic states in the zeroth order scheme. Charge transfer played also an important role in the nonadiabatic molecular dynamics simulations of the protonated Schiff base. In contrast to experiment we found no isomerization. This result is a negative, but we think important one. It should be stressed, that this failure is not introduced by our approximations but already inherent in TDDFT itself.

\section{Acknowledgements}

The authors thank G. Seifert and M. Wanko for fruitful discussion and careful reading of the manuscript. This work is supported by the DFG within the Interdiscipliniary Research Group Molecular Mechanisms of Retinal Protein Action and by the Research Training Group GK-693 of the Paderborn Institute for Scientific Computation (PaSCo). The work of B. Torralva was performed under the auspices of the U. S. Department of Energy by the University of California, Lawrence Livermore National Laboratory under Contract No. W-7405-Eng-48.
[1] A. Zangwill and P. Soven, Phys. Rev. A 21, 1561 (1980).

[2] R. Bauernschmitt and R. Ahlrichs, Chem. Phys. Lett. 256, 454 (1996).

[3] I. Vasiliev, S. Ögüut, and J. R. Chelikowsky, Phys. Rev. Lett. 82, 1919 (1999).

[4] P. L. de Boeij, F. Koostra, J. A. Berger, R. van Leeuwen, and J. G. Sniders, J. Chem. Phys. 1151995 (2001).

[5] F. Furche, R. Ahlrichs, C. Wachsmann, E. Weber, A. Sobanski, F. Vogtle, and S. Grimme, J. Am. Chem. Soc. 1221717 (2000).

[6] K. Yabana, and G. F. Bertsch, Phys. Rev. A 60, 1271 (1999).

[7] D. Heringer, T. A. Niehaus, M. Wanko, and Th. Frauenheim, to be published.

[8] S. J. A. van Gisbergen, J. G. Snijders, and E. J. Baerends, Chem. Phys. Lett. 259, 599 (1996).

[9] S. J. A. van Gisbergen, V. P. Osinga, O. V. Gritsenko, R. van Leeuwen, J. G. Snijders, and E. J. Baerends, J.
Chem. Phys. 105, 3142 (1996).

[10] S. J. A. van Gisbergen, J. G. Snijders, and E. J. Baerends, Phys. Rev. Lett. 78, 3097 (1997).

[11] C. A. Ullrich, S. Erhard, and E. K. U. Gross, in Sper Intense Laser Atom Physics IV, edited by H. G. Muller and M. V. Fedorov, (Kluwer,1996), p. 267-284.

[12] X. Chu and S. I. Chu, Phys. Rev. A 63, 023411 (2001).

[13] A. Castro, M. A. L. Marques, J. A. Alonso, G. F. Bertsch, and A. Rubio, Eur. Phys. J. D 28, 211 (2004).

[14] Y. S. Dou, B. R. Torralva, and R. E. Allen, Chem. Phys. Lett. 392, 352 (2004).

[15] E. Runge and E. K. U. Gross, Phys. Rev. Lett. 52, 997 (1984).

[16] M. E. Casida, in Recent Advances in Density Functional Methods, Part I, edited by D. P. Chong (Singapore, World Scientific, 1995) p. 155.

[17] K. Yabana and G. F. Bertsch, Phys. Rev. B 54, 4484 (1996). 
[18] Here it is assumed, that spin-orbit coupling is neglected.

[19] C. Y. Yam, S. Yokojima, and G. H. Chen, Phys. Rev. B 68, 153105 (2003).

[20] F. Calvayrac, P. G. Reinhard, and E. Suraud, Eur. Phys. J. D 9, 389 (1999); P. G. Reinhard, and E. Suraud, J. Clust. Sci. 10, 239 (1999); E. Suraud, and P. G. Reinhard, Phys. Rev. Lett. 85, 2296 (2000).

[21] T. Brixner, N. H. Damrauer, and G. Gerber, in Adv. in At., Mol. and Opt. Phys. (Academic Press, 2001) Vol. 46, p. 1-54.

[22] R. E. Allen, Phys. Rev. B 50, 18629 (1994).

[23] B. Torralva, T. A. Niehaus, M. Elstner, S. Suhai, Th. Frauenheim, and R. E. Allen, Phys. Rev. B 64, 153105 (2001).

[24] B. R. Torralva and R. E. Allen, J. Mod. Opt. 49, 593 (2002).

[25] Y. S. Dou and R. E. Allen, J. Chem. Phys. 119, 10658 (2003).

[26] Y. S. Dou, B. R. Torralva, and R. E. Allen, J. Mod. Opt. 50, 2615 (2003).

[27] Y. S. Dou, B. R. Torralva, and R. E. Allen, J. Phys. Chem. A 107, 8817 (2003).

[28] Y. S. Dou and R. E. Allen, Chem. Phys. Lett. 378, 323 (2003).

[29] U. Saalmann and R. Schmidt, Z. Phys. D 38, 153 (1996).

[30] U. Saalmann and R. Schmidt, Phys. Rev. Lett. 80, 3213 (1998).

[31] R. Schmidt, O. Knospe, and U. Saalmann, Nuovo Cimento Soc. Ital. Fis. A 110, 1201 (1997).

[32] O. Knospe, J. Jellinek, U. Saalmann, and R. Schmidt, Eur. Phys. J. D 5, 1 (1999).

[33] O. Knospe, J. Jellinek, U. Saalmann, and R. Schmidt, Phys. Rev. A 61, 022715 (2000).

[34] T. Kunert and R. Schmidt, Phys. Rev. Lett. 86, 5258 (2001).

[35] T. Kunert and R. Schmidt, Eur. Phys. J. D 25, 15 (2003).

[36] T. N. Todorov, J.Phys.: Cond. Matt. 1310125 (2001).

[37] T. A. Niehaus, dissertation at the University of Paderborn, http://www.ub.uni-paderborn.de (2001).

[38] T. Frauenheim, G. Seifert, M. Elstner, Z. Hajnal, G. Jungnickel, D. Porezag, S. Suhai, and R. Scholz, phys. stat. sol.(b) 217, 41 (2000).

[39] T. Frauenheim, G. Seifert, M. Elstner, T. Niehaus, C. Köhler, M. Amkreutz, M. Sternberg, Z. Hajnal, A. Di Carlo and S. Suhai, J. Phys.: Cond. Matt. 143015 (2002).

[40] D. Porezag, Th. Frauenheim, Th. Köhler, G. Seifert, R. Kaschner, Phys. Rev. B 51, 12947 (1995).

[41] M. Elstner, D. Porezag, G. Jungnickel, J. Elsner, M. Haugk, Th. Frauenheim, S. Suhai, and G. Seifert, Phys. Rev. B 58, 7260 (1998).

[42] H. M. Polatoglou and M. Methfessel, Phys Rev. B 37, R10403 (1988).

[43] P. Kürpick, W.-D. Sepp, and B. Fricke, Phys. Rev. A 51 3693 (1995).

[44] E. K. U. Gross and W. Kohn, Adv. Quantum Chem. 21, 255 (1990).

[45] M. Graf and P. Vogl, Phys. Rev. B 51, 4940 (1995).

[46] M. A. L. Marques, A. Castro, G. F. Bertsch, and A. Rubio, Comp. Phys. Comm. 151, 60 (2003).

[47] Please note that ionization, which sets in beyond 9.07 eV experimentally [51], can not be correctly described in a localized basis. In this context, a grid representation of the wavefunction together with an implementation of absorbing boundary conditions like in Ref. 17 is more appropriate. We would also like to mention, that the energetical position of the second state around $9 \mathrm{eV}$ is likely to be in error, since this excitation involves a transition to an unbound orbital at the minimal basis level. In spite of these problems, the full spectrum is shown in order to demonstrate the general principle.

[48] C.-P. Hsu, S. Hirata, and M. Head-Gordon, J. Phys. Chem. A 105, 451 (2001).

[49] J. P. Doering, J. Chem. Phys. 70, 3902 (1979).

[50] T. A. Niehaus, S. Suhai, F. Della Sala, P. Lugli, M. Elstner, G. Seifert, and Th. Frauenheim, Phys. Rev. B 63, 085108 (2001).

[51] Ion Energetics Data in NIST Chemistry Webbook, NIST Standard Reference Database Number 69, ed. P.J. Linstrom and W.G. Mallard (National Institute of Standards and Technology, Gaithersburg, MD, 2001) (http://webbook.nist.gov).

[52] D. M. Cox, S. Behal, M. Disko, S. M. Gorun, M. Greaney, C. S. Hsu, E. B. Kollin, J. Millar, J. Robbins, W. Robbins, R. D. Sherwood, and P. Tindall, J. Am. Chem. Soc. 113, 2940 (1991).

[53] H. Ajie, M. M. Alvarez, S. J. Anz, R. D. Beck, F. Dieterich, K. Fostiropoulos, D. R. Huffman, W. Krätschmer, Y. Rubin, K. E. Schriver, D. Sensharma, and R. L. Whetten, J. Phys. Chem. 94, 8630 (1990).

[54] A. Aharoni, B. Hou, N. Friedman, M. Ottolenghi, I. Rousso, S. Ruhman, M. Sheves, T. Ye, and Q. Zhong, Biochemistry (Moscow) 66, 1210 (2001).

[55] H. Kandori, Y. Shichida, and T. Yoshizawa, Biochemistry (Moscow) 66, 1210 (2001).

[56] M. Garavelli, F. Bernardi, M. Olivucci, T. Vreven, S. Klein, P. Celani, and M. A. Robb, Faraday Discuss. 110, 51 (1998).

[57] R. Mathies, and L. Stryer, Proc. Natl. Acad. Sci. USA 73, 2169 (1976).

[58] M. Ponder and R. Mathies, J. Phys. Chem. 87, 5090 (1983).

[59] M. Wanko, M. Garavelli, F. Bernardi, T. A. Niehaus, T. Frauenheim, and M. Elstner, J. Chem. Phys. 120, 1674 (2004).

[60] M. Garavelli, F. Bernardi, M. Robb, and M. Olivucci, Int. J. Photoenergy 4, 57 (2002).

[61] The experimental values from Ref. 62 correspond to similar but not completely equivalent structures. See also the discussion of this point in Ref. 59 .

[62] M. Arnaboldi, M. G. Motto, K. Tsujimoto, V. BaloghNair, and K. Nakanishi, J. Am. Chem. Soc. 101, 7082 (1997); V. Balogh-Nair, J. D. Carriker, B. Honig, V. Kamat, M. G. Motto, K. Nakanishi, R. Sen, M. Sheves, M. Arnaboldi, and K. Tsujimoto, Photochem. Photobiol. 33, 483 (1981); R. F. Chields, G. S. Shaw, and R. E. Wasylishen, J. Am. Chem. Soc. 109, 5362 (1987).

[63] R. Mathies, T. B. Freedman, and L. Stryer, J. Mol. Biol. 109367 (1977).

[64] S. W. Lin, M. Groesbeek, I. van der Hoef, P. Verdegem, J. Lugtenburg, and R. A. Mathies, J. Phys. Chem. 102 2787-2806 (1998).

[65] R. A. Mathies, J. Lugtenburg, in The Primary Photoreaction of Rhodopsin, Handbook of Biological Physics, edited by D. G. Stavenga, W. J. de Grip, and E. N. Pugh Jr., (Elsevier Science B.V., 2000) Vol. 3, Chap. 2.

[66] T. Vreven, F. Bernardi, M. Garavelli, M. Olivucci, M. A. Robb, and H. B. Schlegel, J. Am. Chem. Soc. 119, 12687 
(1997).

[67] S. L. Logunov, L. Song, and M. A. El-Sayed, J. Phys. Chem. 100, 18586 (1996).

[68] A. Dreuw, J. L. Weisman, and M. Head-Gordon, J. Chem. Phys. 119, 2943 (2003).

[69] Y. Tawada, T. Tsuneda, and S. Yanagisawa, J. Chem.
Phys. 120, 8425 (2004).

[70] O. Gritsenko and E.J. Baerends, J. Chem. Phys. 121, 656 (2004).

[71] T. Yanai, D. P. Tew, and N. C. Handy, Chem. Phys. Lett. 393, 51 (2004). 